
Glaubwürdigkeit zurückzukehren! Und nicht selbst genau das sin Reinkultur zu praktizieren, was man meint, der betroffenen Muslima vorhalten zu müssen: zivilisatorische Abgrenzung!

\title{
Mel Kenny \\ Der Fall Pinochet im Dialog zwischen Politik und Justiz
}

Am 24. März 1999 haben die Lordrichter in London ihre zweite Entscheidung über den Antrag der spanischen Regierung, den chilenischen Ex-Diktator Pinochet für Folter, Mord, Geiselnahme und das >Verschwindenlassen « von Menschen innerhalb und außerhalb von Chile während seiner Zeit als Staatsoberhaupt auszuliefern, verkündet ${ }^{1}$. Nachdem der Innenminister am I5. April 1999 die Authority to Proceed erteilt hat, ist die Auslieferung jetzt wieder am Magistrates Court anhängig und wird am 27. September verhandelt. Der Prozeßverlauf hinterläßt einen bitteren Beigeschmack, der die Symbolwirkung und Qualität dieses Präzendenzfalls überschattet.

\section{Politischer Hintergrund}

Am I I. September 1973 fand in Chile ein Staatsstreich statt. Die gewählte Regierung Allende wurde durch eine Militärdiktatur unter General Pinochet ersetzt. Diese Diktatur hielt sich bis zum Rücktritt Pinochets am II. März 1990 an der Macht. Wie Lord Browne-Wilkinson in der zweiten Entscheidung mehr als deutlich machte, war diese Diktatur von einer sentsetzlichen Barbarei< gekennzeichnet. ${ }^{2}$ Über die angewandten Foltermethoden, darunter auch die abwechselnde Folterung von Familienmitgliedern, die in übereinanderliegenden Metallschubladen festgehalten wurden, berichtete am ausführlichsten Lord Steyn in der ersten Entscheidung 3.

Im Oktober 1998 reiste Senator Pinochet zu einer medizinischen Behandlung nach England. Zur gleichen Zeit wurden in Spanien internationale Haftbefehle erlassen, nachdem das Spanische Oberste Gericht die Zuständigkeit der spanischen Gerichte bestätigt hatte. Am 16. und 22. Oktober 1998 sind Haftbefehle von den Richtern (Magistrates) Evans bzw. Bartle in London erstellt worden, worauf Senator Pinochet verhaftet wurde. Die Anwälte Pinochets haben die Aufhebung der Haftbefehle unmittelbar im Queen's Bench Divisional Court beantragt. Die Aufhebung des zweiten

Für eine kritische Durchsicht des Manuskripts danke ich Dr. Andreas Furrer, ZERP, Bremen.

I R v Bartle and the Commissioner of Police for the Metropolis and others Ex Parte Pinochet, R v Evans and Another and the Commissioner of Police for the Metropolis and others Ex Parte Pinochet (On Appeal from a Divisional Court of the Queen's Bench Division), House of Lords, 24 March r 999. Im Website des House of Lords: http://www.parliament.the-stationery-office.co.uk/pa/ld r $99899 / \mathrm{ldjudgmt} / \mathrm{jd} 990324 / \mathrm{pinor}$.htm (Die Internetseiten werden nach 'Teilen (römisch) und Seiten (arabisch) zitiert).

2 ,There is no real dispute that during the period of the Senator Pinochet regime appalling acts of barbarism were committed in Chile and elsewhere in the world: torture, murder and the unexplained disappearance of individuals, all on a large scale. Although it is not alleged that Senator Pinochet himself committed any of those acts, it is alleged that they were done in pursuance of a conspiracy to which he was a party, at his instigation and with his knowledge per Lord Browne-Wilkinson Internet pinor.htm (2 Entscheidung I.,S. 3).

3 R v Bartle and Others ex parte Pinochet [1998] 3 WLR 1456; http://www.parliament.the-stationeryoffice.co.uk/pa/ld 199899/ldjudgmt/jdg8 I I 25/pinoor .htm (IX., S. 3) 
Haftbefehls des Richters Bartle ist aber ausgesetzt worden, während das House of Lords angerufen wurde, um über die seeignete Auslegung und den Umfang der Immunität vor Verbaftung und Auslieferung eines ehemaligen Staatsoberhaupts für Handlungen während seiner Amtszeit zu entscheiden.

\section{Rechtlicher Hintergrund}

Im Verlauf der Entscheidungen im House of Lords wurden vier Fragenkomplexe behandelt:

- Die Frage der Immunität: In diesem Zusammenhang wurden die Fragen der Staatsimmunität, der >Act of State-Doktrin, sowie die Reichweite der Immunität ehemaliger Staatsoberhäupter anhand der Wiener Konvention $1961^{4}$ und des State Immunity Act $1978^{5}$ behandelt.

- Die internationale Konvention gegen Folter und andere grausame, unmenschliche oder erniedrigende Behandlung oder Strafen (Folterkonvention) $1984^{6}:$ Zu überprüfen war der Sinn und Zweck der Konvention, die Auslegung sowohl des Begriffs >Public officials in Artikel I als auch die Reichweite des Verbotes der Folterung in Artikel 2 und vor allem die Interpretation der Artikel $s$ und 6 über die Zuständigkeit der Gerichte und Pflichten zum Tätigwerden.

- Persönlicbe Haftung für Folter: Auf der einen Seite wurde die Frage behandelt, ob Pinochet für die Folterungen persönlich haftbar gemacht werden kann, und auf der anderen Seite, inwieweit eine universelle Gerichtsbarkeit aufgrund der Konkretisierung des internationalen Charakters des Verbots der Folter besteht.

- Die Strafgerichtsbarkeit i.S. des Extradition Act 1989 und die Auswirkungen der Umsetzung der Folterkonvention 1984 im Criminal Justice Act $1988^{7}$. Die in diesem Zusammenhang relevante Frage betraf die Auslegung des Abschnitts 2 (I)(a) des Extradition Act:

2(I) Extradition Act: '... extradition crime means -

(a) conduct in the territory of a foreign state, a designated Commonwealth country or a colony which, if it occurred in the United Kingdom, would constitute an offence punishable...

Letztendlich entschied die Auslegung von if it occurred die Frage, ob sich diese im Zusammenhang mit der Double Criminality Rule auf eine Strafbarkeit in England zur Zeit des Geschehens oder zur Zeit des Auslieferungsgesuchs bezog, den Umfang der strafbaren Handlungen, die eine Auslieferung ermöglichen (Auslieferungsverbrechen, extraclitable offences).

\section{Besonderheiten des englischen Rechts}

Bei der Behandlung dieser Problemkreise sind, aus deutscher Sicht, folgende Besonderheiten des englischen Rechts zu berücksichtigen:

- Erstens ist die Rolle des House of Lords nicht mit der des Bundesverfassungsgerichts vergleichbar, da die Auslieferungsentscheidung letztlich im Ermessen des Innenministers liegt: Dieser übernimmt die politische Abwägung im Einzelfall,

\footnotetext{
4 Wiener Uberetnkommen uber diplomatısche Beztehungen vom 18.4. 1961 BGBl. I 964 II S. 958; UNT'S Vol 500 P. 95

5 The Law Reports, Statutes 1978 I, Ch.33, p.71s.

6 Aus: Resolutionen und Beschlusse der Generalversammlung, Neununddreißigste Tagung, Offizielles Protokoll, Beilage 51, New York 1985, S. 232; GAOR, 39th Session, Resolutions, Suppl. No.5x (UNDoc. A/39/51) p. 197 .

7 Extradition Act 1989, Law Reports, Statutes I989 I 2, Ch.33, p.1747
} 
was Freiräume für eine konsequente juristische Normabwägung durch das House of Lords öffnet. Ursprung dieser Besonderheit ist die Ableitung der Auslieferungskompetenz aus den ungeschriebenen königlichen Vorrechten. Damit unterscheiden sich die involvierten verfassungsrechtlichen Instanzen nicht nur in ihrer Rolle, sondern auch in der Breite der eingeräumten exekutiven Befugnisse. Dies wirkt sich auf das Verständnis der Souveränität in den beiden Ländern aus.

- Zweitens müssen internationale Abkommen im dualistischen England für ihre Anwendbarkeit in nationales Recht umgesetzt werden.

- Drittens muß eine Rückwirkung eines Gesetzes explizit geregelt sein.

Unter Berücksichtigung dieser Besonderheiten ist die unterschiedliche Gewichtung und Stellung der Problemkreise in den zwei Entscheidungen von besonderer Bedeutung. Diese werden daher in aller Kürze getrennt dargestellt.

\section{Die erste Entscheidung des House of Lords - Umfang der Immunität und die Frage, ob Senator Pinochet persönlich verantwortlich sei ${ }^{8}$}

Nicht nur die Brisanz des Falles, sondern auch die Eigentümlichkeit der im House of Lords praktizierten Form der Urteilsverkündung - jeder Richter gibt der Reihe nach seine Stellungnahme ab, und es entscheidet die Mehrheit - sorgten für Spannung. Nachdem die Lords Slynn und Lloyd aufgrund der Feststellung, daß die Handlungen Pinochets zu den Funktionen eines Staatsoberhaupts gehörten, dafür plädierten, daß Pinochet die Immunität beanspruchen könne, haben die Lords Nicholls, Steyn und Hoffmann die Immunität Pinochets abgelehnt. Laut Lord Nicholls konnte er sich weder auf die Staatsimmunität, da diese nicht den Einzelnen schützt, noch auf die persönliche Immunität i.S. der Wiener Konvention, da diese nur die durch internationales Recht annerkannten Handlungen schützt, berufen'.

Darüber hinaus entschied die Mehrheit, daß die >Act of State-Doktrin, die eine Zuständigkeit nur in Chile begründet hätte, keine Anwendung fand. Am ausführlichsten führte Lord Steyn diesen Punkt aus: Erstens sei es nicht die Aufgabe des House of Lords, über die Inhalte des Haftbefehls oder die Schuld Pinochets zu entscheiden. Zweitens habe der Gesetzgeber nicht beabsichtigt, über die >Act of State<-Doktrin eine Immunität für Folter zuzulassen. Drittens finde die >Act of State<Doktrin keine Anwendung, sobald der Gesetzgeber eine Gerichtsbarkeit über die Handlungen anderer Staaten etabliert habe - entweder durch die Transformation völkerrechtlicher Verträge bezüglich `Folter^ im Abschnitt ${ }_{34}$ Criminal Justice Act 1988, infolge der Folterkonvention 1984 oder durch das Einführen von neuen Straftaten wie im Abschitt 1 Taking of Hostages Act 1982. Letztlich finde die Doktrin keine Anwendung, da die Handlungen bereits 1973 als Straftaten i.S. des internationalen Gewohnheitsrechts anerkannt gewesen seien.

Abschließend wurde auch eine >Restimmunität` für Senator Pinochet verneint. Diese sei nur in den von Lord Nicholls aufgezeichneten Grenzen der >Act of Stater-Doktrin zulässig und dürfe nicht soweit gehen, daß sie die Existenz sowohl völkerrechtlich als auch national anerkannter Verbrechen in Frage stelle. In ähnlicher Weise verneinte auch Lord Steyn die Immunität Pinochets, wobei er die Schwäche der Behauptung, $\mathrm{da} ß$ Pinochet nicht persönlich verantwortlich sei, hervorhob ${ }^{10}$. Am kürzesten faßte

8 Siehe oben Fn. 3, auch K. Ambos, Der Fall Pinochet und das anwendbare Recht, JZ 1999, I6.

9 .... it hardly needs sayıng that torture of his own subjects... would not be regarded by international law as a function of a head of state. per Lord Nicholls (i Entscheidung VIII., S. 6).

10... this ... flies in the face of an elementary principle of law, shared by all civilized legal systems, that there is no distinction to be drawn between the man who strikes, and a man who orders another to strike.< per Lord Steyn ( I Entscheidung IX., S. 6). 
sich Lord Hoffmann: II have...(read) in draft the speech of my noble and learned friend Lord Nicholls of Birkenhead and for the reasons be gives I too would allow this appeal. ${ }^{11}$ Nach dieser 3:2-Mehrheitsentscheidung gegen die Immunität Pinochets hat der Innenminister der zuständigen Magistrate am 9. Dezember 1998 die Erlaubnis erteilt, mit dem Auslieferungsverfahren (Authority to proceed) unter Abschnitt 7 des Extradition Act 1989 fortzufahren.

\section{Befangenheit - und die Frage, ob Lord Hoffmann persönlich voreinge- nommen war}

Nachdem bekannt wurde, daß Lady Hoffmann Mitarbeiterin und Lord Hoffmann Direktor von Amnesty International Charity Limited waren und damit eng mit der Geschäftsführung von Amnesty International (AI), einer Organisation, die auch als Streithelfer zugelassen wurde, verbunden waren, reichten die Anwälte Pinochets am 10. Dezember 1998 einen Antrag auf Aufhebung der Entscheidung des House of Lords bzw. auf Ungültigerklärung des Votums von Lord Hoffmann ein. Zuvor hatte die Verteidigung am 7. Dezember 1998 den Innenminister von der möglichen Voreingenommenheit des Richters unterrichtet. Dieser zog aber keine Konsequenzen daraus und erteilte die Authority to Proceed.

Diesem Antrag wurde einstimmig stattgegeben. ${ }^{\mathrm{I}}$ Die Anwälte Pinochets machten, auch wenn nicht ohne Ironie, zu Recht geltend, es handele sich bei den Verbindungen Lord Hoffmanns zu AI nicht um die Frage, ob Lord Hoffmann tatsächlich voreingenommen sei, sondern ob der Eindruck entstehen könne, daß er Richter in eigener Sache gewesen sei. Die Lordrichter machten deutlich, daß weder finanzielle Interessen im Spiel seien, noch daß Lord Hoffmann tatsächlich voreingenommen gewesen sei. Allein seine Führungsrolle bei AI reiche aus, um ihn automatisch von der Richterbank auszuschließen. Die Rolle von Lady Hoffmanns als Mitarbeiterin und Lord Hoffmanns bei der Eintreibung von Spenden wären nur bedeutsam gewesen, wenn Pinochet eine konkrete Gefahr oder eine angemessene Vermutung der Voreingenommenheit hätte beweisen müssen ${ }^{13}$. Darüber hinaus betonten die Lordrichter, daß nur Entscheidungen, die auf der Basis eines Verfahrensfehlers getroffen worden seien, aufgehoben werden können: Es genüge nicht, wenn im nachhinein Entscheidungen für >nicht passend ‘ gefunden würden. Auch die Behauptung der Staatsanwaltschaft, durch die Intervention der Anwälte Pinochets beim Innenminister habe ein Mißbrauch des Prozeßverfahrens stattgefunden, wurde von den Lords zurückgewiesen: nur wenn die Verteidigung von der möglichen Befangenheit eines Richters vor Urteilsverkündung gewußt hätte, wäre eine Frage des Mißbrauchs des Prozeßverfahrens aufzuwerfen gewesen. Auch wurde von den Lords unterstrichen, daß der Innenminister nach dem 25 . November 1998 keine Alternative zur Erteilung der Authority to Proceed hatte.

Einstimmig wurde die erneute Verhandlung der Sache im House of Lords entschieden. Aber nur Lord Browne-Wilkinson nahm explizit Stellung zur Begründung dieser Entscheidung: Es solle den Parteien erspart bleiben, vor einer Richterbank aufzutreten, wo vier der Meinungen schon bekannt seien. ${ }^{14}$ Angesichts der Tatsache, daß in

I 1 Entscheidung IX. S. 8.

I2 In $r e$ Pinochet [1999] 2 W. L. R. 272; http://www.parliament.the-stationery-office.co.uk/pa/ldig9899/ Idjudgmt/jdg9o s s/pinoor.htm.

13 per Lord Browne-Wilkinsons (Befangenheitsantrag I., S. 10).

I 4 It was appropriate to direct a re-hearing of the appeal before a differently constituted Committee, so that on the re-hearing the parties were not faced with a Committee four of whom had already expressed their conclusion on the points of law at issue per Lord Brown-Wilkinson (Befangenheitsantrag I., S. I I). 
diesem Fall das Votum von Lord Hoffmann für die 3:2-Entscheidung ausschlagebend war, ist diese Argumentation nachvollziehbar, aber kritikwürdig: Nur implizit deutete Lord Goff den grundsätzlichen Vorrang einer Neuverhandlung auch in Fällen an, wo die Mehrheiten anders gelagert sind's.

\section{Die Neuverhandlung im House of Lords}

a) Die Strafgerichtsbarkeit->Double Criminality، (beiderseitige Strafbarkeit) als Filter für die Immunitätsanalyse

Die zweite Entscheidung beschränkte ihre Aussagen: Dabei gaben die Lordrichter der Frage den Vorrang vor der Immunitätsfrage: Diese wurde nur auf die Fälle von Auslieferungsverbrechen i.S. des Extradition Act 1989 erstreckt. Nach der Double Criminality rule konnten nur Verbrechen Gegenstände der Immunitätsanalyse sein, die sowohl in England als auch, wie in diesem Fall, in Spanien strafbar waren. Da die Folterkonvention 1984 erst am 29. September 1988 im Criminal Justice Act umgesetzt worden ist und dieses Gesetz keine Rückwirkungsklausel enthält, war die Entscheidung, ob ein >Auslieferungsverbrechen ein Verbrechen in beiden Ländern zur Zeit des Auslieferungsgesuchs oder zur Zeit des Geschehens sein mußte, von zentraler Bedeutung. In diesem Zusammenhang wurde die Auslegung des zweiten Abschnitts des Extradition Act 1989 vorgenommen.

Entgegen der Entscheidung sowohl des Lord Chief Justice im Divisional Court als auch der Zugeständnisse in der ersten Entscheidung des House of Lords fand das Gericht die wortwörtliche Auslegung des Abschnitts doppeldeutig: if it occurred könne sich sowohl auf den Zeitpunkt des Geschehens als auch des Gesuchs beziehen. Im Kontext sowohl des Abschnitts 9(8) des Extradition Act 1989, der festlegt, daß der Magistrate insofern von der Strafbarkeit überzeugt sein muß, als die Handlungen had occurred (erfolgt sind), als auch des ersten Schedules des Extradition Act I $870,{ }^{16}$ stellte das House of Lords fest, daß die Strafbarkeit zur Zeit des Geschehens ausschlaggebend sei. Nach dieser Entscheidung bleiben nur wenige der angeklagten Verbrechen und keine aus dem unmittelbaren Umfeld des Sturzes der Allende-Regierung - die jedoch die brisantesten Fälle sind - sondern nur Folter und Verschwörung zur Folter nach dem 29. September 1988 sowie Verschwörung zum Mord und Folter in Spanien übrig. Darüber hinaus fand das Gericht die Fälle der Geiselnahme für nicht verhandelbar, da nur die Geiseln und nicht Dritte, wie im Taking of Hostages Act 1982 festgelegt, zu Handlungen oder Unterlassungen gezwungen werden sollten.

\section{b) Persönliche Haftung für Folter}

Nach der restriktiven Eingrenzung der verhandelbaren Fälle stellten die Lordrichter die persönliche Haftung eines Staatsoberhaupts fest. Nach der Errichtung des Nürnberger Tribunals sei nicht nur die persönliche Haftung für Verbrechen im internationalen Recht anerkannt worden, sondern diese sei auch aus ihrer Abhängigkeit von der Kriegführung herausgelöst worden. Hierbei wurde auf die UN-Resolutionen 3059, 3452 und 3453 sowie auf die Einrichtung von internationalen Tribunalen für Rwanda und für das ehemalige Jugoslawien Bezug genommen. ${ }^{17}$ Im internationalen

is >I would add that in any case where the impartiality of a judge is in question the appearance of the matter is just as important as the realitye (Befangenheitsantrag II.S. 3).

I6 I Schedule, Extradition Act 1870: 'The following list of crimes is to be construed according to the law existing in England... at the date of the alleged crimer.

17 Statute of the International Criminal Tribunal for Yugoslavia (Artikel 7 (2) ILM 32 (1993)p. I 192); Statute for the Criminal Tribunal for Rwanda (Artikle 6 (2) ILM 33(r994) p.1602) 
Recht habe das Verbot der Folter den übergeordneten Charakter des ius cogens. Dies hat zur Folge, daß sie den Staaten eine universelle Gerichtsbarkeit liefert, wie dies in den Fällen Attorney-General of Israel $v$ Eichmann ${ }^{18}$ und Demjanjuk $v$ Petrovsky unterstrichen wurde: Folterer gelten heute als >common enemies of all mankind and all nations have an equal interest in their apprehension and prosecution. ${ }^{19}$

\section{c) Die Folterkonvention}

Nach Browne-Wilkinson besteht das Problem, das durch die Folterkonvention bekämpft werden sollte, darin, daß es keine internationalen Gerichte gibt, die für dieses Verbot zuständig sind, und daß die Verfolgung der Verantwortlichen im eigenen Land politisch ungewollt bzw. dank einer Amnestie ausgeschlossen sein könnte. Sinn der Folterkonvention, so die Lordrichter, ist es, Folterern den ssicheren Hafen zu nehmen; sie sollen sich nicht mehr den rechtlichen Konsequenzen ihrer Handlungen durch einem Grenzüberschritt entziehen können.

Die Fragen, die die Folterkonvention aufwarf, betrafen den Status von General Pinochet als Staatsoberhaupt und der Zuständigkeit der Gerichte. Zur ersten Frage wurde im House of Lords eindeutig klargemacht, daß General Pinochet als Staatsoberhaupt ein spublic official i.S. des Artikel r der Konvention darstellt. Alles andere hätte gegen Sinn und $Z$ weck der Konvention verstoßen und die Statusfrage mit der Frage der Immunität vermengt. Zur zweiten Frage wurde auf das in der Konvention verbriefte Prinzip aut dedere aut punire verwiesen und damit die universelle Gerichtsbarkeit betont. Nach den Artikeln 2, 4, 5, 6 und 8 der Konvention mußte England entweder das Verfahren eröffnen oder ausliefern.

\section{d) Immunität}

Zur Beurteilung der kritischen Frage der Immunität haben die Lordrichter, wie in der ersten Entscheidung, die Wiener Konvention über diplomatische Beziebungen 1961 und dabei insbesondere den Artikel 39 der Konvention über die Immunität eines ausscheidenden Botschafters, der im Abschnitt 20(I) des State Immunity Act 1978 die diplomatic privileges auf Staatsoberhäupte ausdehnt, berücksichtigt. Damit wurde unterschieden zwischen der Immunität ratione materiae, wonach für seine offiziellen Amtshandlungen die Immunität aufrechterhalten bleibt, und der Immunität ratione personae, wonach die persönliche Immunität nach der Amtszeit verlorengeht. Zu den Amtshandlungen können internationale Straftaten, Handlungen gegen die Menschheit und ius cogens nicht gehören. Für Amtshandlungen, die kriminell waren, aber nicht gegen ius cogens verstießen, wurde dagegen Immunität zuerkannt, vorausgesetzt, daß auf diese im Herkunftsland nicht verzichtet wurde. ${ }^{20}$ Für rein private Handlungen ist keine Immunität geboten. ${ }^{2 x}$ Anders könne man die Immunität nicht auslegen, sonst würde man die Großen schützen und die Kleinen hängen. ${ }^{22}$

18 Attorney-General of Israel v Eichmann [1962] 36 ILR s.

I9 Demjanjuk v Petrovsky [1985] 603 F. Supp. 1468; 776 F 2d. 571 .

20 Marcos and Marcos v Federal Department of Police [1989] I02 ILR I98.

21 Unted States $v$ Nonega [1990] 746 F. Supp 1506.

22 3... if the former head of state has immunity, the man most responsible will escape liability while his inferiors who carried out his orders will be liable. I find it impossible to accept that this was the intention per Lord Browne-Wilkinson (2 Entscheidung II., S. 6). 
Der Prozeßverlauf: Der nachträgliche Antrag auf Befangenheit, die daraus folgende Aufhebung der ersten Entscheidung des House of Lords und die, auf eine neue Argumentation aufbauende zweite Entscheidung, die damit implizierte Kritik sowohl an der Methodik des Lord Chief Justice im Divisional Court als auch an der ersten Richterbank im House of Lords, ist alles andere als zufriedenstellend. An manchen Stellen entsteht sogar der bedauerliche Eindruck, daß es mehr um die >mögliche< Befangenheit eines Richters oder die implizite Unfähigkeit einer Richterbank im House of Lords gehe, als daß die persönliche Verantwortung eines Exdiktators für die rentsetzliche Barbarei`während seiner Herrschaft den Gegenstand des Verfahrens darstellt. Auch die in der zweiten Entscheidung erfolgte Reduzierung des Umfangs der Auslieferungsverbrechen hinterläßt einen bitteren Beigeschmack. Dazu kommt, daß mehrere Lordrichter den Innenminister ermahnten, den Fall nochmals kritisch zu bewerten.

Die Problematik der Entscheidung konzentriert sich auf die Frage, ob die vorgenomme Abwägung zwischen den erwähnten Normen und die daraus resultierende Reduzierung des Umfangs der >A uslieferungsverbrechen « richtig und zwingend notwendig waren. In diesem Zusammenhang sind $\mathrm{m}$. E. zwei ineinander verstrickte Argumente, die die Kohärenz der zweiten Entscheidung gefährden, hervorzuheben:

- In der zweiten Entscheidung stellte das House of Lords fest, daß der Begriff if it occurred doppeldeutig sei. Selbst wenn dies zutrifft (was aber bezweifelt werden kann), ist wohl eine Auslegung im Lichte der neueren Entwicklungen des Völkerrechts seit dem Nürnberger Tribunal eher angemessen als die vorgenommene pragmatische und historische Auslegung i.S. des Extradition Act 1870 .

- Die Folterkonvention hat keinen >neuen $<$ Straftatbestand im internationalen Recht geschaffen. Lord Hutton betonte zu Recht, daß das Verbot von Folter die Geltung als ius cogens bereits vor der Umsetzung der Folterkonvention in England hatte. Er argumentierte weiter, daß den Richtern aber keine Zuständigkeit für Auslieferungsverbrechen vor dem Inkrafttreten des Criminal Justice Act zukommt: Seine weitere Schlußfolgerung, daß daher die Frage, wann Folter vom internationalen Recht als Verbrechen anerkannt wurde, überflüssig werde, erscheint aber wenig überzeugend und äußerst pragmatisch ${ }^{23}$. Konsequenter erscheint die Linie Lord Savilles ${ }^{24}$, daß die Folterkonvention bereits existierende Verbrechen um einzelne Handlungen erweitert und ausgedehnt hat, um die bis dahin notwendige enge sinstitutionelle< Dimension der Folter zu überwinden ${ }^{25}$. Darüber hinaus hat die Folterkonvention die Pflicht zum Tätigwerden aut dedere aut punire eingeführt ${ }^{26}$ : Vor der Folterkonvention hätten Staaten die Möglichkeit gehabt, tätig zu werden; nach der Konvention müßten sie es werden. Leider konnte Lord Saville keine Mehrheit für diese Position gewinnen.

$23 \times$ As your Lorships hold that there is no jurisdiction to extradite Senator Pinochet for Acts of torture prior to 29 September $1988 \ldots$ it is unnecessary to decide when torture became a crime against international lawa per Lord Hutton (2 Entscheidung VI., S. s).

24 .The systematic use of torture... had joined piracy... as an international crime of universal jurisdiction well before 1984 . I consider that it had done so by 1973 . For my own part... I would hold that the courts of this country already possessed extra-territorial jurisdiction... on the scale of the charges in the present case and did not require the authority of statute to exercise it per Lord Saville (2 Entscheidung VIII., S. I/ 2).

25 . Whereas the international community had condemned the widespread and systematic use of torture... the Convention extended the offence to cover isolated and individual instances of torcure provided that they were committed by a public official.< per Lord Saville (2 Entscheidung VIII., S. 2).

26 . Whereas previously states were entitled to take jurisdiction... they were now placed under an obligation to do so. Any state... in whose territory a person... was found was bound to offer to extradite him or to initiate proceedings to prosecute him.`per Lord Savile (2 Entscheidung VIII., S. 2). 
Im Lichte dieser Kritikpunkte zeigt sich die pragmatische Seite der zweiten Entscheidung. Auffällig ist es, daß die zeitlich gezogene Grenze der Immunität im Einklang mit dem Ende des Kalten Krieges und der Nützlichkeit Pinochets sowohl im Kampf der Systeme als auch für England im Streit um die Falkland-Inseln steht. Die Problematik bezüglich der Staatsoberhäupter, die man einst hofiert hat und denen man im nachhinein den Prozeß macht, ist aber gerade in diesen Tagen aktueller denn je. Darüber hinaus haben die Lordrichter immer wieder betont, daß sie auf die politischen Konsequenzen in Chile keine Rücksicht nehmen könnten. Über die Konsequenzen in England und die Beliebtheit des Landes als Urlaubsziel unter >Vietnam Vets sollte aber genauso wenig Rücksicht genommen werden - zumindest erscheint der Preis für die weitere Rechtsentwicklung hierfür etwas zu hoch.

Nachdem der Innenminister jetzt die zweite $>$ Authority to Proceed erteilt hat und die Anwälte Pinochets ihre Absicht, den Rechtsweg auszuschöpfen, bekanntgegeben haben, ist zu erwarten, daß Pinochet noch lange in England bleiben wird: Gegen die mögliche Auslieferungsentschiedung des Magistrates Court kann jeweils zuerst im Divisional Court und dann im House of Lords Berufung eingelegt werden. Die endgültige Genehmigung der Auslieferung durch den Innenminister kann dann schießlich vor dem House of Lords nochmals angefochten werden. Dabei muß betont werden, daß die endgültige Entscheidung des Innenministers nicht durch die Vorverfahren determiniert ist.

Der Fall ist ein Beweis dafür, wie schwer sich der Rechtsstaat mit den schwierigsten der allerschwersten Fälle tut. Gleichwohl werden gerade hier die Unterschiede zwischen den entsetzlichen Methoden eines Pinochet und der beschwerlichen Suche nach juristischen Antworten, die in solchen Fällen nie zufriedenstellend sein können, deutlich. Der Fall entwickelt dadurch eine hohe Symbolwirkung an die wahren Adressaten in Serbien, Kroatien und Ruanda.

Über diese Symbolwirkung hinaus ist die Entscheidung ein Präzedenzfall: Zum ersten Mal ist der Antrag eines Staatsoberhaupts auf Immunität im Zusammenhang mit Verbrechen gegen die Menschlichkeit zurückgewiesen worden: die sicheren Häfen sind genommen worden ${ }^{27}$. Hinzu kommt, daß mit der Anerkennung der universellen Gerichtsbarkeit aut dedere aut punire eine Handhabe gegen Folterer geschaffen wurde, die vom Zögern nationaler Gerichte oder der erteilten nationalen Amnestie unabhängig ist (auch, wie in Südafrika, von >Truth Commissions< erteilt?). Letztlich ist ein erster praxisrelevanter Schritt getan worden, der vom noch mit vielen Unsicherheiten behafteten Bemühen um die Errichtung internationaler Gerichte unabhängig ist.

27 In future those who commit atrocities against civilian populations must expect to be called to account if fundamental human rights are to be properly protected. In this context, the exalted rank of the accused can afford no defence.c per Lord Saville (2 Entscheidung VIII., S. 4). 